\title{
13. TRACE ELEMENTS: FRACTIONAL CRYSTALLIZATION AND PARTIAL MELTING PROCESS, HETEROGENEITY OF UPPER MANTLE MATERIAL
}

\author{
H. Bougault and P. Cambon, Centre Oceanologique de Bretagne, B.P. 337, 29273, Brest Cedex, France \\ and \\ L. Joron and M. Treuil, Groupe des Sciences de la Terre, ${ }^{1}$ Laboratoire Pierre Sue, C.E.N. Saclay, B.P. $\mathrm{n}^{\circ} 2,91190$ \\ Gif-sur-Yvette, France
}

\section{INTRODUCTION}

From a study of trace elements, including first transition series elements and low parition coefficient elements or so-called hygromagmatophyle elements (Treuil and Varet, 1973; Treuil and Joron, 1976), we will try to establish the three fundamental parameters (mantle material, partial melting, and crystallization) which influence the formation of basalts recovered during Leg 46. Data are presented in connection with shipboard study; a tentative interpretation is given taking into account our present knowledge of the behavior of trace elements during magmatic processes and comparing the results of FAMOUS, Leg 37 and Leg 45. We ask the reader to look at our Leg 45 report (Bougault, et al., 1978 ) to gain information about analytical procedures and geochemical methods of investigation.

\section{DESCRIPTION OF LEG 46 RESULTS}

Several chemical units were defined onboard (Shipboard Scientific Party, this volume). Downhole they correspond to three aphyric units (A1, A2, and A3), then two phyric units (B1 and B2) with a final aphyric unit (C). All samples (except 396B-19-1, 4-6 cm) were chosen for being as fresh as possible. The last aphyric sample (396B-32-1, 45-47 cm) is different from Unit $C$. As expected, the difference between aphyric and plagioclase phyric samples is clearly shown by $\mathrm{Al}_{2} \mathrm{O}_{3}$ and $\mathrm{CaO}$; only slight differences (Table 1, average and sigma values) can be observed within aphyric units or within phyric units except for $\mathrm{TiO}_{2}$, which is the best discriminant among major or minor elements because of its low partition coefficient.

Trace element concentrations expressed in ppm, including $\mathrm{Ni}, \mathrm{Cr}, \mathrm{Zr}$, and $\mathrm{Sr}$ which were measured onboard, are reported in Table 2. $\mathrm{Ti}, \mathrm{Mn}$, and $\mathrm{Fe}$ expressed in ppm are included to have the full first transition series (except Sc), all elements being classified as a function of increasing atomic number. All the trace elements measured (especially low partition coefficient [LPC] hydromagmatophile elements such as $\mathrm{Zr}, \mathrm{Eu}, \mathrm{Tb}, \mathrm{Hf}, \mathrm{Ta}$, and $\mathrm{Th}$ ) confirm the classification and the definition of chemical units which were made onboard. Table 3 shows concentrations and sigma values of investigated elements for each unit. Since there is no change

'Laboratoire associe au C.R.S. 196; Laboratoire de Geochimie et Cosmochimie; Institut de Physique du Globe, Universite Paris VI, 4 Place Jussieu, 75230 Paris, Cedex 05.
TABLE 1

Major Element Average Values for Each Unit in Hole 396B

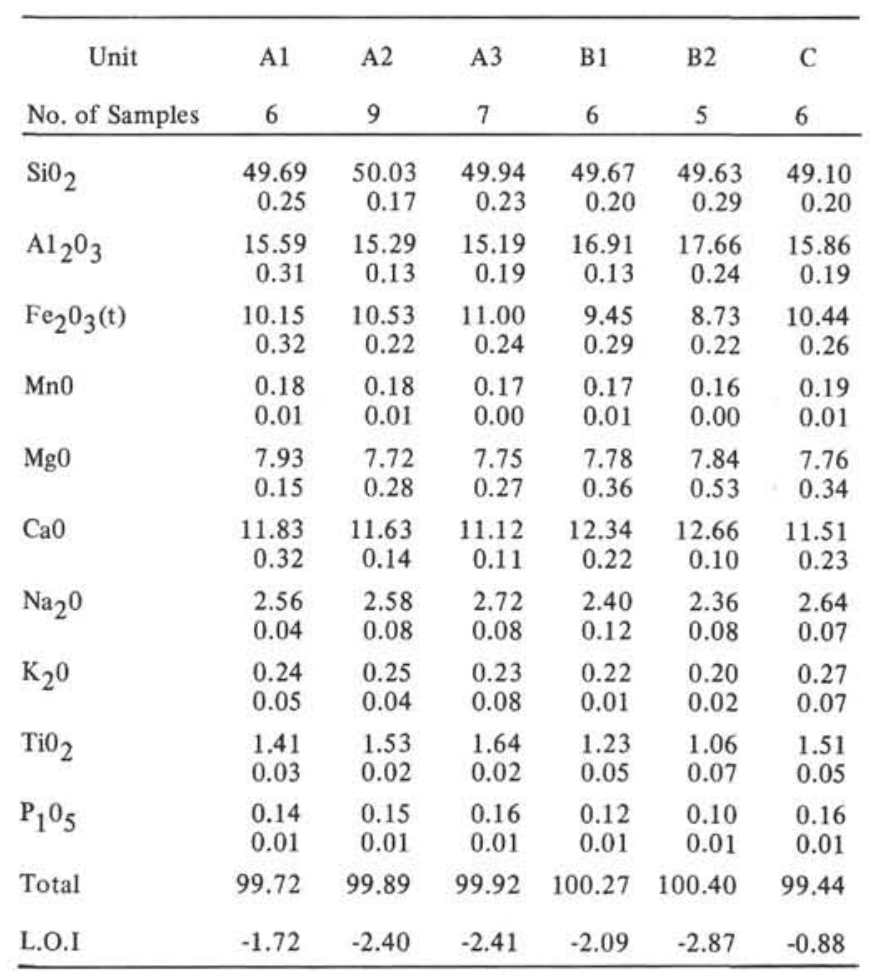

in the definition of chemical units, we refer the reader to the Site Report (this volume) for complete descriptions of each unit.

$\mathrm{Co}, \mathrm{Cu}$, and $\mathrm{Zn}$ concentrations are remarkably constant. This can now be considered a well-established fact for oceanic tholeiites. A general correlation between V and LPC elements including Ti can be observed confirming that $\mathrm{V}$ has a partition coefficient lower than one; but $\mathrm{V}$ cannot be really considered as a true LPC element because this correlation has already been proved to be not very good and among this data an inversion is observed between Unit A2 $(\mathrm{Ti}=9160 \pm 99$; $\mathrm{V}=293 \pm 5)$ and $\mathrm{A} 3(\mathrm{Ti}=9822 \pm 113 ; \mathrm{V}=282 \pm 5)$. Sr varies between 126 to $145 \mathrm{ppm}$ for Units Al, A2, A3, B1, B2, and is a little higher in Unit C (i.e., $156 \mathrm{ppm}$ ). Highest $\mathrm{Sr}$ values are not observed in plagioclase phyric units $(B 1=133 \mathrm{ppm}$, B2 $=135 \mathrm{ppm}$ ) as was the case in Hole 395A.

It can be observed that Unit $\mathrm{A} 3$ is homogeneous with respect to all analyzed elements except for $\mathrm{K}_{2} \mathrm{O}$ (which is 
TABLE 2

Trace Element Concentrations (ppm) in Hole 396B

\begin{tabular}{|c|c|c|c|c|c|c|c|c|c|c|c|c|c|c|c|c|c|c|c|c|}
\hline \multirow{2}{*}{$\begin{array}{c}\text { Sample } \\
\text { (Interval in } \mathrm{cm} \text { ) }\end{array}$} & \multirow{2}{*}{$\begin{array}{c}\mathrm{Ti} \\
\text { XRF }\end{array}$} & \multirow{2}{*}{$\begin{array}{l}v \\
\text { XRF }\end{array}$} & \multirow{2}{*}{$\begin{array}{l}\mathrm{Cr} \\
\mathrm{XRF}\end{array}$} & \multirow{2}{*}{$\begin{array}{l}\mathrm{Mn} \\
\mathrm{XRF}\end{array}$} & \multirow{2}{*}{$\begin{array}{l}\mathrm{Fe} \\
\mathrm{XRF}\end{array}$} & \multicolumn{3}{|c|}{ Co } & \multicolumn{2}{|c|}{ N1 } & \multirow{2}{*}{$\begin{array}{r}\mathrm{Cu} \\
\mathrm{AA}\end{array}$} & \multirow{2}{*}{$\begin{array}{l}\mathrm{Zn} \\
\mathrm{AA}\end{array}$} & \multirow{2}{*}{$\begin{array}{l}\mathrm{Sr} \\
\text { XRF }\end{array}$} & \multicolumn{2}{|c|}{$\mathrm{Z}_{\mathrm{r}}$} & \multirow{2}{*}{$\begin{array}{l}\mathrm{Eu} \\
\mathrm{NA}\end{array}$} & \multirow{2}{*}{$\begin{array}{l}\mathrm{Tb} \\
\mathrm{NA}\end{array}$} & \multirow{2}{*}{$\begin{array}{l}\mathrm{H} 1 \\
\mathrm{NA}\end{array}$} & \multirow{2}{*}{$\begin{array}{l}\mathrm{Ta} \\
\mathrm{NA}\end{array}$} & Th \\
\hline & & & & & & XRF & AA & $\mathrm{NA}$ & XRF & NA & & & & $\mathrm{XRF}$ & NA & & & & & NA \\
\hline $4-1,103-105(=9)$ & 8600 & 238 & 275 & 1394 & 71400 & 41 & 45 & 46 & 146 & 146 & 66 & 80 & 126 & 97 & 104 & 1.25 & 0.75 & 2.50 & 0.176 & 0.125 \\
\hline $5-1,86-88(\# 9)$ & 8200 & 266 & 306 & 1394 & 70070 & 40 & 45 & 41 & 124 & 128 & 57 & 77 & 118 & 89 & 110 & 1.21 & 0.74 & 2.44 & 0.169 & 0.140 \\
\hline $5-2,51-53( \pm 6 A)$ & 8400 & 282 & 313 & 1316 & 71470 & 42 & 41 & 43 & 140 & 141 & 67 & 80 & 120 & 95 & 97 & 1.29 & 0.73 & 2.52 & 0.176 & 0.120 \\
\hline $6-1,55-57(\# 7)$ & 8600 & 250 & 278 & 1316 & 67060 & 39 & 41 & 45 & 138 & 142 & 67 & 80 & 146 & 90 & 110 & 1.26 & 0.73 & 2.52 & 0.176 & 0.105 \\
\hline $7-1,53-55(=7)$ & 8520 & 272 & 297 & 1471 & 73290 & 41 & 41 & 43 & 133 & 134 & 66 & 80 & 122 & 99 & 92 & 1.25 & 0.72 & 2.49 & 0.175 & 0.136 \\
\hline $7-1,132-134(\# 11)$ & 8520 & 288 & 319 & 1394 & 72940 & & 41 & 44 & 133 & 143 & 66 & 80 & 124 & 96 & 106 & 1.20 & 0.72 & 2.38 & 0.169 & 0.120 \\
\hline $8-1,62-64(\# 8)$ & 9060 & 291 & 313 & 1471 & 74270 & 43 & 41 & 43 & 124 & 131 & 62 & 78 & 131 & 92 & 95 & 1.34 & 0.76 & 2.56 & 0.185 & 0.155 \\
\hline $8-2,60-62(\# 6)$ & 9300 & 303 & 282 & 1471 & 72030 & 43 & 41 & & 130 & & 67 & 80 & 141 & 109 & & & & & & \\
\hline $10-8,51-53(\# 9 \mathrm{~A})$ & 9420 & 295 & (364) & 1549 & 76300 & 43 & 41 & 43 & 120 & 128 & 68 & 82 & 128 & 111 & 104 & 1.34 & 0.81 & 2.67 & 0.194 & 0.136 \\
\hline $11-1,56-58( \pm 6)$ & 9240 & 295 & 279 & 1549 & 74970 & 41 & 41 & 43 & 120 & 128 & 63 & 84 & 126 & 103 & 120 & 1.42 & 0.78 & 2.56 & 0.185 & 0.114 \\
\hline $11-2,5-7(=1)$ & 9240 & 291 & 277 & 1394 & 74270 & 44 & 41 & 44 & 131 & 128 & 64 & 88 & 132 & 103 & 108 & 1.36 & 0.78 & 2.76 & 0.187 & 0.150 \\
\hline $12-1,127 \cdot 129(\# 8)$ & 9060 & 295 & 281 & 1394 & 73430 & 45 & 45 & 44 & 126 & 137 & 64 & 77 & 136 & 99 & 105 & 1.28 & 0.75 & 2.70 & 0.182 & 0.148 \\
\hline $13-1,45-47( \pm 4)$ & 9180 & 285 & 275 & 1394 & 74550 & 41 & 41 & 42 & 131 & 132 & 65 & 80 & 124 & 100 & 98 & 1.35 & 0.76 & 2.68 & 0.182 & 0.115 \\
\hline $13-2,49-51(\# 5)$ & 9060 & 297 & 278 & 1316 & 71820 & 46 & 41 & 44 & 138 & 138 & 61 & 76 & 130 & 99 & 112 & 1.25 & 0.74 & 2.60 & 0.179 & 0.140 \\
\hline $13-2,89-91(\approx 10)$ & 9060 & 288 & 278 & 1316 & 72030 & 43 & 41 & 46 & 145 & 144 & 63 & 84 & 144 & 94 & 100 & 1.35 & 0.76 & 2.65 & 0.182 & 0.112 \\
\hline $13-3,4-6(\# 1)$ & 10020 & 287 & 255 & 1316 & 73990 & 40 & 45 & 48 & 156 & 165 & 63 & 88 & 146 & 126 & 121 & 1,48 & 0.89 & 3.04 & 0.207 & 0.14 \\
\hline $14-2,17 \cdot 19(\approx 2)$ & 9840 & 283 & 269 & 1316 & 75670 & 45 & 45 & 45 & 155 & 154 & 65 & 75 & 143 & 129 & 141 & 1.43 & 0.86 & 2.88 & 0.19 & 0.123 \\
\hline $15-1,85-87(\# 1)$ & 9900 & 272 & 260 & 1316 & 77060 & 46 & 41 & 45 & 150 & 147 & 63 & 84 & 146 & 119 & 132 & 1.43 & 0.87 & 2.98 & 0.192 & 0.156 \\
\hline $15-2.129-131(\# 2)$ & 9780 & (235) & (237) & 1316 & 78400 & 38 & 41 & 42 & 119 & 132 & 63 & 80 & 142 & 124 & 121 & 1.43 & 0.85 & 2.90 & 0.194 & 0.147 \\
\hline $15-3,16-19(=2 B)$ & 9780 & 287 & 277 & 1316 & 77280 & 43 & 41 & 42 & 119 & 133 & 63 & 82 & 140 & 116 & 100 & 1.43 & 0.84 & 2.80 & 0.189 & 0.155 \\
\hline $15-4,76-79(\# 4)$ & 9780 & 283 & 268 & 1394 & 78050 & 43 & 41 & 43 & 116 & 138 & 63 & 80 & 142 & 117 & 118 & 1.52 & 0.85 & 3.10 & 0.20 & 0.160 \\
\hline $15-5,70-73(\# 9)$ & 9660 & 282 & 263 & 1394 & 78610 & 45 & 41 & 44 & 149 & 152 & 64 & 86 & 156 & 111 & 130 & 1.46 & 0.85 & 3.03 & 0.196 & 0.160 \\
\hline $16-1,83-85(=10)$ & 7200 & 238 & 301 & 1239 & 64470 & 39 & 37 & 41 & 129 & 138 & 65 & 74 & 127 & 78 & 89 & 1.12 & .64 & 2.08 & 0.138 & 0.104 \\
\hline $16-2,40-42(+4 A)$ & 7200 & 296 & 317 & 1316 & 66150 & 42 & 41 & 43 & 155 & 163 & 69 & 71 & 133 & 77 & 86 & 1.03 & 0.59 & 2.01 & 0.135 & 0.090 \\
\hline $16-4 \cdot 20-22(\neq 2)$ & 7980 & 271 & 316 & 1394 & 69860 & 42 & 41 & 42 & 118 & 122 & 69 & 77 & 130 & 83 & 98 & 1.20 & 0.70 & $2 ; 29$ & 0.157 & 0.094 \\
\hline $16-5,96-98(\# 11)$ & 7260 & 248 & 301 & & 64330 & 39 & 41 & 42 & 133 & 138 & 66 & 81 & 135 & 81 & 90 & 1.09 & 0.63 & 2.15 & 0.139 & 0.082 \\
\hline $17-1,132-134(=\mathrm{H} 11 \mathrm{~B})$ & 7260 & 245 & 315 & 1316 & 66640 & 44 & 41 & 43 & 144 & 149 & 69 & 71 & 142 & 79 & 92 & 1.10 & 0.61 & 2.14 & 0.136 & 0.12 \\
\hline $18-1,117-119(\# 7 D)$ & 7260 & 244 & 307 & 1316 & 65520 & 40 & 37 & 40 & 119 & 125 & 63 & 71 & 131 & 81 & 90 & 1.10 & 0.62 & 2.16 & 0.137 & 0.11 \\
\hline $19-1.4-6(\# 1)$ & 7980 & 282 & 306 & 1471 & 73220 & 41 & 49 & 44 & 102 & 106 & 73 & 80 & 143 & 88 & 93 & 1.21 & 0.68 & 2.39 & 0.153 & 0.12 \\
\hline $20-1,53-55(\neq 4 B)$ & 6240 & 238 & 311 & 1239 & 61670 & 39 & 37 & 40 & 119 & 128 & 65 & 71 & 148 & 74 & 84 & 1.01 & 0.58 & 1.98 & 0.133 & 0.098 \\
\hline $20-3,33-35(\# 4)$ & 6600 & 217 & 350 & 1239 & 60830 & 39 & 45 & 41 & 139 & 148 & 70 & 65 & 141 & 66 & 78 & 0.97 & 0.53 & 1.85 & 0.118 & 0.106 \\
\hline $20-5,16 \cdot 18(\# 2)$ & 6960 & 226 & 342 & 1239 & 63420 & 43 & 45 & 41 & 139 & 146 & 73 & 63 & 144 & 73 & 76 & 0.97 & 0.57 & 1.87 & 0.122 & 0.084 \\
\hline $21-2,24-26(\# 2)$ & 6060 & 212 & 342 & 1160 & 60130 & 41 & 37 & 41 & 157 & 166 & 71 & 70 & 124 & 68 & 56 & 0.98 & 0.53 & 1.70 & 0.116 & 0.091 \\
\hline $22-1.93-95(\# 11)$ & 5940 & 180 & 342 & 1239 & 59430 & 44 & 37 & 41 & 154 & 162 & 70 & 64 & 121 & 63 & 62 & 0.89 & 0.52 & 1.62 & 0.113 & 0.066 \\
\hline $23-1.87-89(=12)$ & 9480 & 284 & 305 & 1394 & 74064 & 39 & 41 & 40 & 140 & 139 & 65 & 78 & 163 & 106 & 123 & 1.40 & 0.79 & 2.86 & 0.322 & 0.33 \\
\hline $24-1,94-96(\# 15)$ & 9240 & 257 & 282 & 1394 & 73150 & 41 & 41 & 41 & 133 & 149 & 65 & 77 & 161 & 109 & 113 & 1.88 & 0.75 & 2.55 & 0.315 & 0.28 \\
\hline $26-1.7-10(\# 1)$ & 8940 & 289 & 272 & 1471 & 72310 & 42 & 37 & 42 & 139 & 144 & 61 & 78 & 157 & 115 & 136 & 1.30 & 0.76 & 2.55 & 0.307 & 0.218 \\
\hline $30-1.60$ & 8940 & 275 & 298 & 1471 & 76020 & 39 & 41 & 40 & 128 & 138 & (116) & 75 & 156 & (80) & 133 & 1.24 & 0.76 & 2.54 & 0.259 & 0.200 \\
\hline Basaltic Sand & & & & & & & & & & & & & & & & & & & & \\
\hline $30-1.60$ & 9000 & & & 1471 & 71890 & & 41 & & 137 & & 66 & 82 & 150 & 103 & & & & & & \\
\hline Glass of Sand & & & & & & & & & & & & & & & & & & & & \\
\hline $32-1.45 \cdot 47(\# 7)$ & 8700 & 266 & 301 & 1394 & 70910 & 41 & 41 & 41 & 122 & 130 & 67 & 72 & 154 & 108 & 121 & 1.28 & 0.74 & 2.50 & 0.271 & 0.178 \\
\hline $32-1,69-71(\neq 10)$ & 7320 & 230 & 322 & 1084 & 57610 & 39 & 41 & 38 & 155 & 164 & 61 & 64 & 162 & 85 & 95 & 0.92 & 0.62 & 2.07 & 0.226 & 0.180 \\
\hline
\end{tabular}

TABLE 3

Trace Element Average Values (ppm) for Each Unit in Hole 396B

\begin{tabular}{|c|c|c|c|c|c|c|c|c|c|c|c|c|c|c|c|c|c|c|c|c|}
\hline Unit & $\underset{\text { XRF }}{\mathrm{Ti}}$ & $\begin{array}{c}\mathrm{V} \\
\mathrm{XRF}\end{array}$ & $\begin{array}{c}\mathrm{Cr} \\
\mathrm{XRF} \\
\end{array}$ & $\underset{\mathrm{Mn}}{\mathrm{MnF}}$ & $\begin{array}{c}\mathrm{Fe} \\
\text { XRF } \\
\end{array}$ & XRF & $\begin{array}{l}\text { Co } \\
\mathrm{AA} \\
\end{array}$ & NA & XRF $^{\mathrm{N}}$ & NA & $\begin{array}{l}\mathrm{Cu} \\
\mathrm{AR} \\
\end{array}$ & $\begin{array}{l}\mathrm{Zn} \\
\mathrm{AR} \\
\end{array}$ & $\begin{array}{c}\mathrm{Sr} \\
\mathrm{XRF}\end{array}$ & XRF & NA & $\begin{array}{r}\mathrm{Eu} \\
\mathrm{NA} \\
\end{array}$ & $\begin{array}{r}\mathrm{Tb} \\
\mathrm{NA} \\
\end{array}$ & $\begin{array}{l}\mathrm{Hf} \\
\mathrm{NA}\end{array}$ & $\begin{array}{l}\mathrm{Ta} \\
\mathrm{NA} \\
\end{array}$ & $\begin{array}{l}\text { Th } \\
\text { NA } \\
\end{array}$ \\
\hline $\begin{array}{c}A 1 \\
\begin{array}{c}\bar{x} \\
s\end{array}\end{array}$ & $\begin{array}{r}8473 \\
153\end{array}$ & $\begin{array}{r}266 \\
19\end{array}$ & $\begin{array}{r}298 \\
18\end{array}$ & $\begin{array}{r}1380 \\
58\end{array}$ & $\begin{array}{r}70920 \\
2168\end{array}$ & $\begin{array}{r}40.6 \\
1.1\end{array}$ & $\begin{array}{r}42.3 \\
2.1\end{array}$ & $\begin{array}{r}43.7 \\
1.7\end{array}$ & $\begin{array}{c}136 \\
7.5\end{array}$ & $\begin{array}{l}139 \\
6.7\end{array}$ & $\begin{array}{r}65 \\
4\end{array}$ & $\begin{array}{r}79.4 \\
1.2\end{array}$ & $\begin{array}{r}126 \\
10\end{array}$ & $\begin{array}{r}94 \\
4\end{array}$ & $\begin{array}{c}103 \\
7.3\end{array}$ & $\begin{array}{l}1.24 \\
0.03\end{array}$ & $\begin{array}{l}0.73 \\
0.01\end{array}$ & $\begin{array}{l}2.47 \\
0.05\end{array}$ & $\begin{array}{l}0.174 \\
0.004\end{array}$ & $\begin{array}{l}0.124 \\
0.01\end{array}$ \\
\hline $\begin{array}{r}\mathrm{A} 2 \\
\bar{x} \\
\mathrm{~s}\end{array}$ & $\begin{array}{r}9160 \\
89\end{array}$ & $\begin{array}{r}293 \\
5\end{array}$ & $\begin{array}{r}282 \\
12\end{array}$ & $\begin{array}{r}1428 \\
87\end{array}$ & $\begin{array}{r}73741 \\
1537\end{array}$ & $\begin{array}{l}43 \\
1.6\end{array}$ & $\stackrel{41}{1.3}$ & $\begin{array}{r}43.5 \\
1.3\end{array}$ & $\begin{array}{r}129 \\
8\end{array}$ & $\begin{array}{l}133 \\
5.5\end{array}$ & $\begin{array}{l}64 \\
2.2\end{array}$ & $\begin{array}{l}81 \\
3.8\end{array}$ & $\begin{array}{r}132 \\
6\end{array}$ & $\begin{array}{r}101 \\
6\end{array}$ & $\begin{array}{r}104 \\
7\end{array}$ & $\begin{array}{l}1.35 \\
0.04\end{array}$ & $\begin{array}{l}0.76 \\
0.02\end{array}$ & $\begin{array}{l}2.65 \\
0.006\end{array}$ & $\begin{array}{l}0.182 \\
0.005\end{array}$ & $\begin{array}{l}0.136 \\
0.016\end{array}$ \\
\hline $\begin{array}{r}\mathrm{A} 3 \begin{array}{r}\bar{x} \\
s\end{array} \\
\end{array}$ & $\begin{array}{r}9822 \\
113\end{array}$ & $\begin{array}{r}282 \\
5\end{array}$ & $\begin{array}{c}265 \\
7.7\end{array}$ & $\begin{array}{r}1338 \\
38\end{array}$ & $\begin{array}{r}77010 \\
1661\end{array}$ & $\begin{array}{r}43 \\
1\end{array}$ & $\begin{array}{c}42 \\
0.7\end{array}$ & $\begin{array}{l}44 \\
0.8\end{array}$ & $\begin{array}{l}118 \\
1.7\end{array}$ & $\begin{array}{l}134 \\
3.2\end{array}$ & $\stackrel{63}{0.8}$ & $\begin{array}{l}82 \\
4.3\end{array}$ & $\begin{array}{l}145 \\
5.3\end{array}$ & $\begin{array}{c}120 \\
6.3\end{array}$ & $\begin{array}{r}123 \\
13\end{array}$ & $\begin{array}{l}1.45 \\
0.03\end{array}$ & $\begin{array}{l}0.86 \\
0.017\end{array}$ & $\begin{array}{l}2.96 \\
0.10\end{array}$ & $\begin{array}{l}0.195 \\
0.006\end{array}$ & $\begin{array}{l}0.149 \\
0.013\end{array}$ \\
\hline $\begin{array}{c}B 1 \\
\bar{x} \\
s\end{array}$ & $\begin{array}{r}7360 \\
305\end{array}$ & $\begin{array}{r}257 \\
22\end{array}$ & $\begin{array}{l}309 \\
7.5\end{array}$ & $\begin{array}{r}1316 \\
54\end{array}$ & $\begin{array}{r}66161 \\
2025\end{array}$ & $\begin{array}{r}41 \\
2\end{array}$ & $\begin{array}{r}39 \\
2\end{array}$ & $\begin{array}{l}42 \\
1.2\end{array}$ & $\begin{array}{r}133 \\
14\end{array}$ & $\begin{array}{r}139 \\
15\end{array}$ & $\begin{array}{l}67 \\
2.5\end{array}$ & $\begin{array}{r}74 \\
4\end{array}$ & $\begin{array}{r}133 \\
5\end{array}$ & $\begin{array}{l}80 \\
2.2\end{array}$ & $\begin{array}{r}91 \\
4\end{array}$ & $\begin{array}{l}1.10 \\
0.05\end{array}$ & $\begin{array}{l}0.63 \\
0.038\end{array}$ & $\begin{array}{l}2.14 \\
0.09\end{array}$ & $\begin{array}{l}0.14 \\
0.008\end{array}$ & $\begin{array}{l}0.100 \\
0.014\end{array}$ \\
\hline $\begin{array}{c}B 2 \underset{x}{\bar{x}} \\
s\end{array}$ & $\begin{array}{r}6360 \\
417\end{array}$ & $\begin{array}{r}214 \\
21\end{array}$ & $\begin{array}{r}337 \\
15\end{array}$ & $\begin{array}{r}1223 \\
34\end{array}$ & $\begin{array}{r}61096 \\
1541\end{array}$ & $\begin{array}{r}41 \\
2\end{array}$ & $\begin{array}{r}40 \\
4\end{array}$ & $\begin{array}{c}41 \\
0.4\end{array}$ & $\begin{array}{r}141 \\
15\end{array}$ & $\begin{array}{r}150 \\
15\end{array}$ & $\begin{array}{r}70 \\
3\end{array}$ & $\begin{array}{l}67 \\
3.6\end{array}$ & $\begin{array}{r}135 \\
12\end{array}$ & $\begin{array}{l}69 \\
4.6\end{array}$ & $\begin{array}{l}71 \\
11.7\end{array}$ & $\begin{array}{l}0.96 \\
0.04\end{array}$ & $\begin{array}{l}0.52 \\
0.03\end{array}$ & $\begin{array}{l}1.8 \\
0.14\end{array}$ & $\begin{array}{l}0.12 \\
0.008\end{array}$ & $\begin{array}{l}0.089 \\
0.015\end{array}$ \\
\hline $\begin{array}{ll}C & \bar{x} \\
& s\end{array}$ & $\begin{array}{r}9050 \\
272\end{array}$ & $\begin{array}{r}276 \\
13\end{array}$ & $\begin{array}{r}291 \\
14\end{array}$ & $\begin{array}{r}1432 \\
42\end{array}$ & $\begin{array}{r}730566 \\
1806\end{array}$ & $\begin{array}{l}40 \\
1.3\end{array}$ & $\begin{array}{l}40 \\
1.6\end{array}$ & $\begin{array}{l}41 \\
0.8\end{array}$ & $\begin{array}{r}133 \\
7\end{array}$ & $\begin{array}{r}140 \\
7\end{array}$ & $\stackrel{65}{2.3}$ & $\begin{array}{c}77 \\
3.3\end{array}$ & $\begin{array}{r}156 \\
4.7\end{array}$ & $\begin{array}{r}108 \\
4.4\end{array}$ & $\begin{array}{r}125 \\
9\end{array}$ & $\begin{array}{l}1.30 \\
0.06\end{array}$ & $\begin{array}{l}0.76 \\
0.02\end{array}$ & $\begin{array}{l}2.6 \\
0.14\end{array}$ & $\begin{array}{l}0.295 \\
0.03\end{array}$ & $\begin{array}{l}0.22 \\
0.04\end{array}$ \\
\hline
\end{tabular}

significant because it is alternation-dependent) and $\mathrm{Ni} . \mathrm{Ni}$ values for Samples 396B-15-2, 179-181 cm and 396B-15-3, $16-18 \mathrm{~cm}$ to $396 \mathrm{~B}-15-4,76-79 \mathrm{~cm}$ are lower (both XRF and NA data) than other samples of Unit A3. Olivine abundance within the unit is lower than 1 per cent, and no significant variation of phenocrysts is mentioned.

Unit $\mathrm{C}$ is not homogeneous in respect of $\mathrm{Th}, \mathrm{Ta}$, and $\mathrm{Hf}$ and shows the highest values for these elements.

\section{DISCUSSION AND INTERPRETATION OF RESULTS}

From the shipboard study, we received information on $\mathrm{Ti}$ and $\mathrm{Zr}$ concentrations. These elements plot on the same straight line passing through the origin as Leg 37 and 45 samples (Figure 1). It has been mentioned (Bougault et al., 1978) that aphyric samples have higher $\mathrm{TiO}_{2}$ and $\mathrm{Zr}$ than 


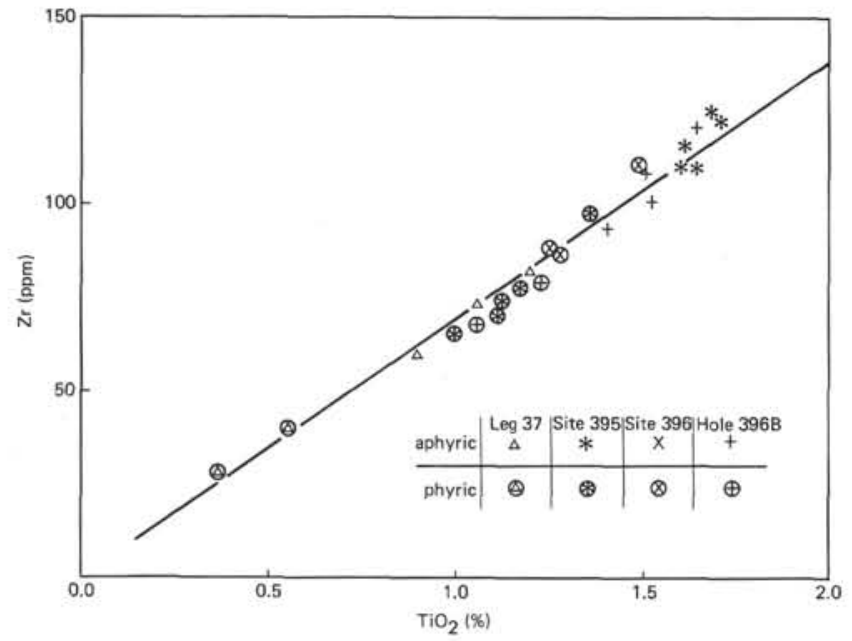

Figure 1. Zirconium versus titanium $\left(\mathrm{TiO}_{2}\right)$, comparison of results from Legs 45 and 37. Note a shift along the line from Leg 37 to Leg 45 with samples for phyric samples on one hand and aphyric on the other.

phyric samples. This can be attributed to a difference of partial melting range and a different initial mantle material.

Leg 46 samples plot in same ranges as Leg 45 samples both for aphyric and phyric samples. This suggests a similarity between Holes 396, 396B, and those at Site 395 (which are symmetric to Site 396 with respect to the Mid-Atlantic Ridge). This suggests that similar conclusions can be derived from Hole 396B (Leg 56) as they were from Holes 395 and 396 (Leg 45) with regards to the nature of the upper mantle material at $20^{\circ} \mathrm{N}$ compared with $36^{\circ} \mathrm{N}$ (FAMOUS).

Hygromagmatophyle elements with low partition coefficients $\mathrm{Ta}, \mathrm{Hf}, \mathrm{Tb}, \mathrm{Zr}$, and $\mathrm{Ti}$ have been plotted as a function of Th in Figures 2, 3, 4, 5 and 6. FAMOUS samples are also presented on these figures. Leg 46 samples plot on the same straight line passing through the origin as Leg 45 samples confirming the above-mentioned conclusion for Sites 395 and 396 . It can be noted that Unit C does not plot on the same line; it is the only unit showing this feature among Leg 45 and 46 results. We do not know if this can be attributed to the possibility of partial melting of a different material at a same site on the ridge, or to another effect. We mention again that this unit shows the maximum of dispersion for LPC elements and that sand and gravels are present in this unit.

The following are the main conclusions of this study regarding Leg 45 LPC elements: (1) the ratio of one LPC element to another LPC element is the same for Sites 395 and 396 (symmetric with respect to the MAR), (2) these ratios differ between $20^{\circ} \mathrm{N}$ (Leg 45 and 46) and $36^{\circ} \mathrm{N}$ (FAMOUS), implying a different nature of the initial upper mantle material melted at these latitudes.

Log $\mathrm{Cr}$ versus $\log \mathrm{Ni}$ is plotted in Figure 7, where Leg 37 and FAMOUS sample fields (aphyric and phyric) are mentioned, as well as Leg 45 data. Leg 46 units plot in a narrow range on the FAMOUS aphyric trend, with a similar slope, except one of the two subunits of A3.

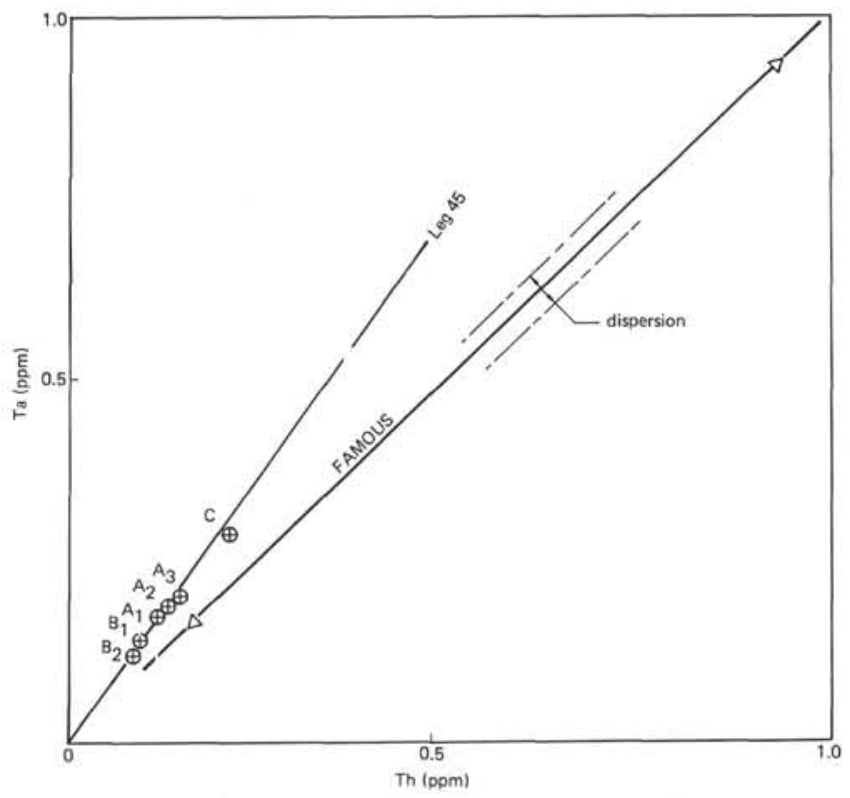

Figure 2. Tantalum versus thorium, a low partition coefficient (LPC) hygromagmatophyle element as a function of a very low LPC hygromagmatophyle element, showing upper mantle heterogeneity (difference between Legs 45 and 46 on one hand and FAMOUS on the other). (Note that samples from Holes 396, 396 A, 396, and 396B plot on the same line.)

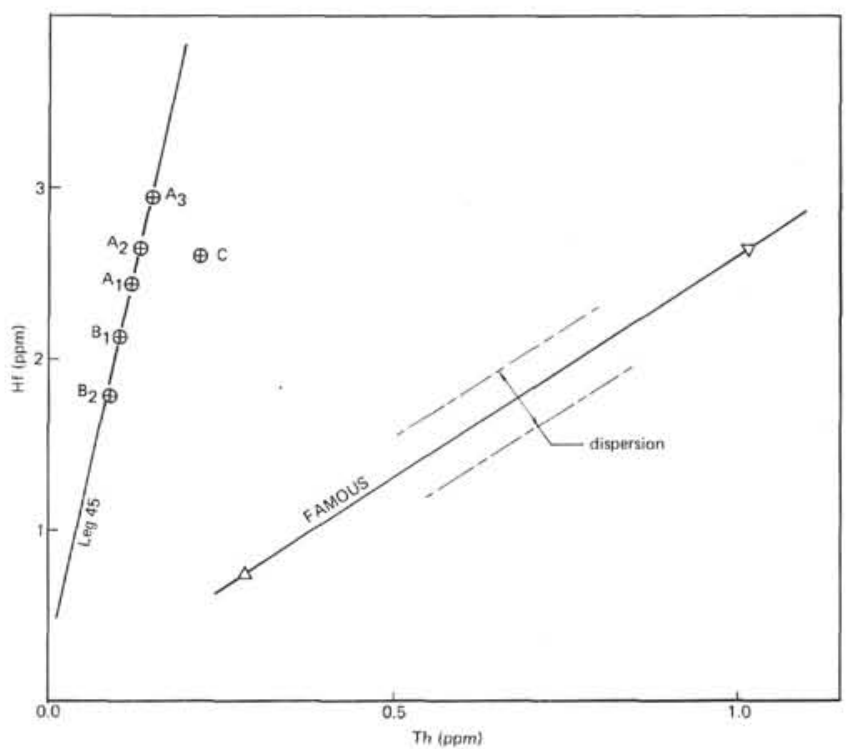

Figure 3. Hafnium versus thorium, a LPC element as a function of a very low LPC element, showing upper mantle heterogeneity of variable partial melting. All units except Unit C plot on the same line as Leg 45 samples.

All units can be classified in the same way: increasing LPC elements, and decreasing $\mathrm{Ni}$ and $\mathrm{Cr}$. This suggests that these basalts were derived from melts produced by similar partial melting. It is conceivable that B1 derives from B2. It 


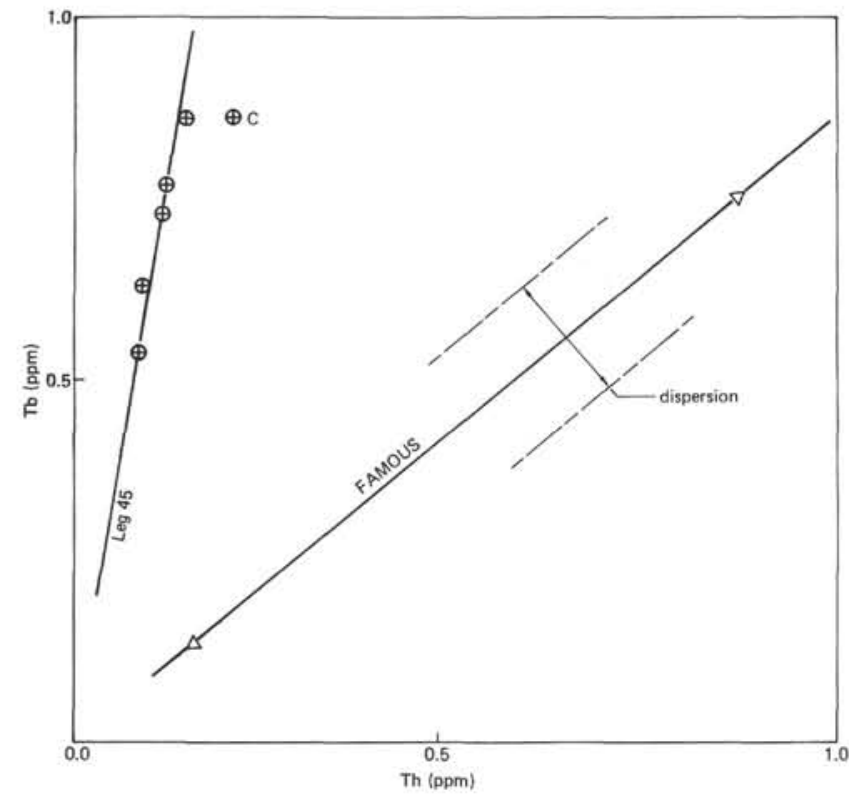

Figure 4. Terbium versus thorium, same information as Figure 3.

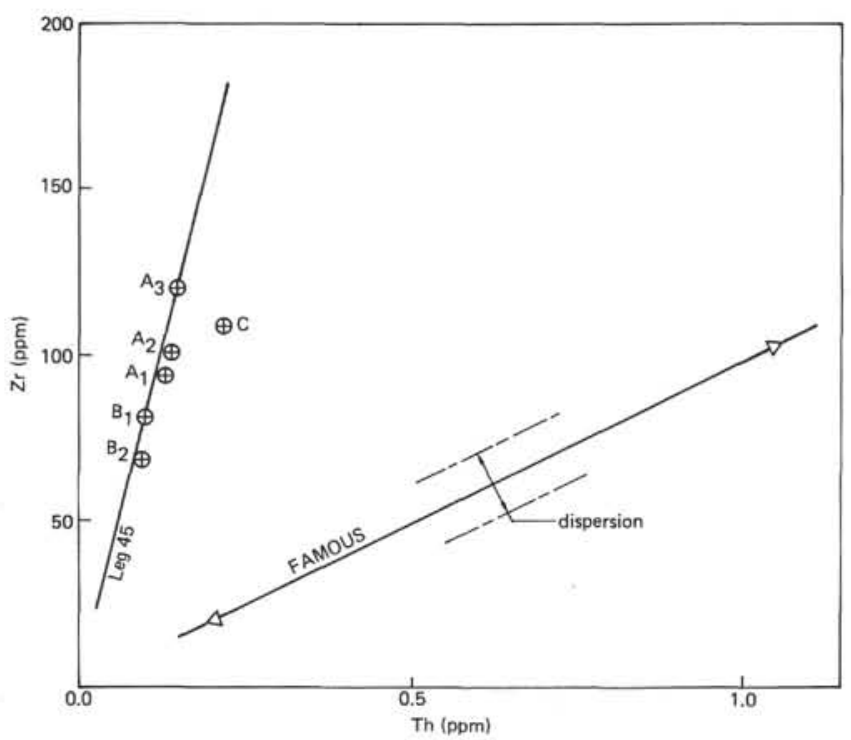

Figure 5. Zirconium versus thorium, same information as Figure 3.

is more difficult to make $\mathrm{A} 1, \mathrm{~A} 2$, and $\mathrm{A} 3$ derive one from each other because of their stratigraphy (as the less evolved unit would be at the top).

\section{CONCLUSION}

Trace elements data allow classification of samples recovered during Leg 46 , following the same units obtained from major elements by the shipboard study.

From high partition coefficient nd ttw partition coefficient elements, the following can be concluded: (1) none of the basaltic samples recovered can be considered as primary liquids, (2) all have undergone a substantial history of

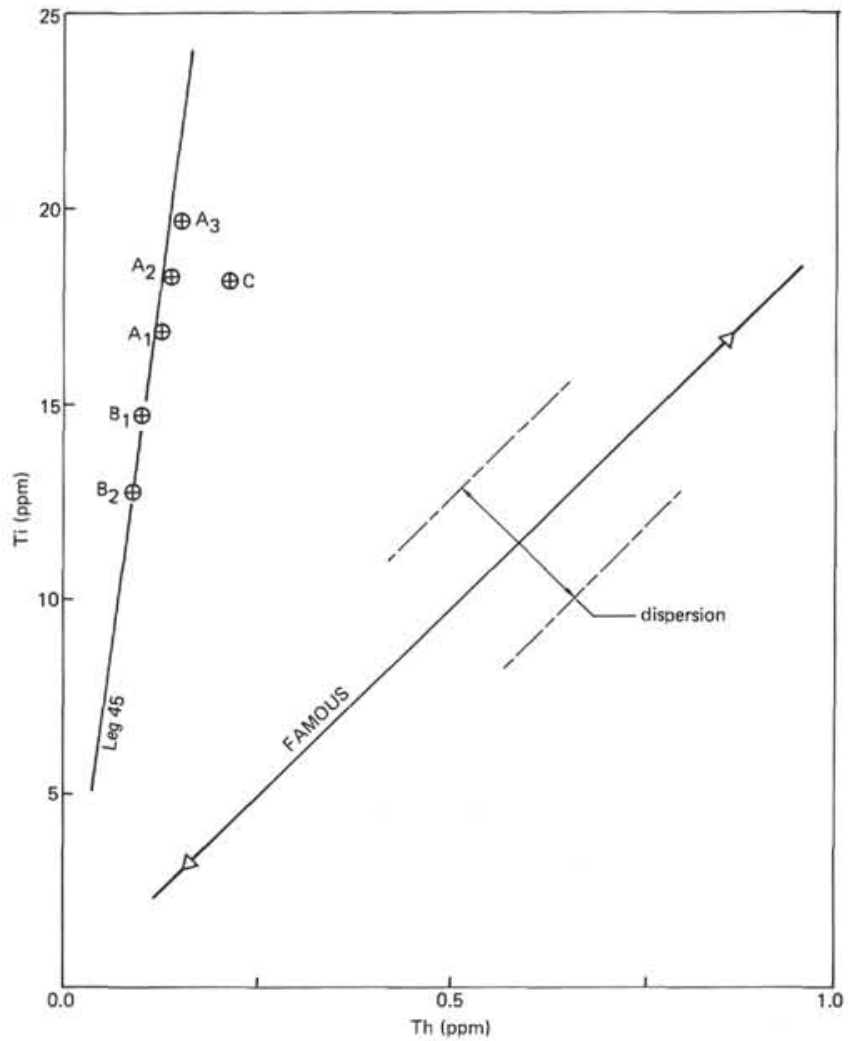

Figure 6. Titanium versus thorium, same information as Figure 3.

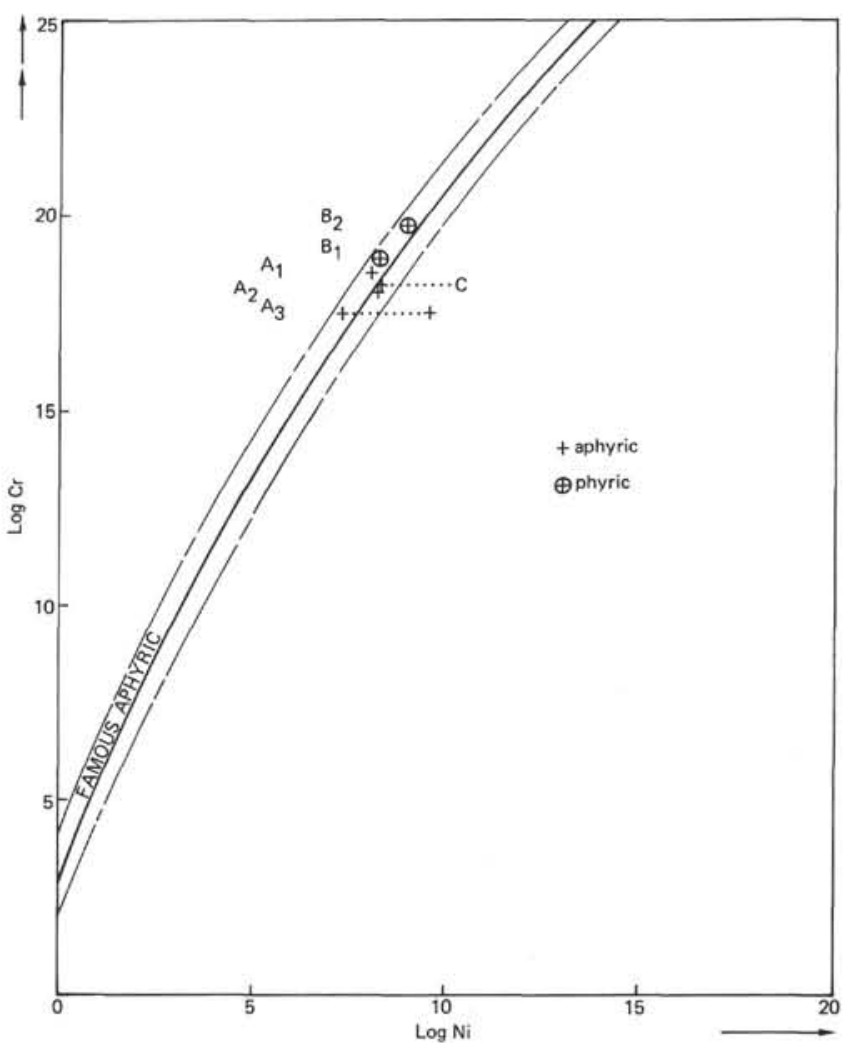

Figure 7. $\operatorname{Leg}(\mathrm{Cr})$ versus $\log (\mathrm{Ni})$. 
crystallization, and (3) they can be derived from melts produced by similar partial melting.

Low partition coefficient hygromagmatophyle elements confirm Hole 396 data. Material found at Sites 395 and 396 (symmetric with respect to the MAR) are derived from similar initial mantle material. The initial mantle material at $20^{\circ} \mathrm{N}$ (Legs 45 and 46 ) is different from initial mantle material at $36^{\circ} \mathrm{N}$ (FAMOUS area).

\section{REFERENCES}

Treuil, M. and Joron, L., 1976. Etude geochimique des elements en trace dans le magmatisme de l'AFAR. Implication pretogenetique et comparaison avec le magmatisme de l'Islande et de la dorsale medio atlantique. Inter-union or an international symposium on AFAR region and related tift problems held in Bad Bergrabern F.R. Germany, 1-6 April 1976, v. II, E. Scherbart'she Verlagsbuchhanalung (Nugle U. Obermiller), Stuttgart, 1976.

Treuil, M. and Varet, M., 1973. Criteres volcanologiques et geochimiques de la genese et de la differentiation des magmas basaltiques: exemple de l'AFAR, Bull. Soc. Geol., France, v. 15 , p. $506-540$.

Bougault, H., Treuil, M., and Joron, L., 1978. Trace elements: fractional crystallization and partial melting processes, heterogeneity of the upper mantle material, Leg 45. In Melson, W.G., Rabinowitz, P.D., et al., Initial Reports of Deep Sea Drilling Project, v. 45: Washington (U.S. Government Printing Office). 